\title{
HIGH-FIDELITY MULTI-PHYSICS PIN-BY-PIN MODEL OF A SVEA-96 OPTIMA2 ASSEMBLY WITH TORT-TD/CTF
}

\author{
A. Seubert, Y. Périn, R. Henry \\ Gesellschaft für Anlagen- und Reaktorsicherheit (GRS) gGmbH \\ Boltzmannstr. 14, D-85748 Garching, Germany \\ armin.seubert@grs.de, yann.perin@grs.de, romain.henry@grs.de
}

\begin{abstract}
This paper describes the development of a 3-d high-fidelity multi-physics pin-by-pin neutronic and sub-channel thermal hydraulic model of a SVEA-96 Optima2 fuel assembly for the coupled code system TORT-TD/CTF. No pin power reconstruction is applied. Detailed descriptions are given on (a) the generation of few-group parameterized pin cellhomogenized cross section data using HELIOS, (b) the 3-d neutronic pin-by-pin and thermal-hydraulic sub-channel model of a single SVEA-96 Optima2 assembly and its qualification by Monte Carlo models using Serpent, (c) coupled steady-state results for a single assembly and (d) coupled steady-state and transient simulations of a 4-by-4 mini core model of SVEA-96 Optima2 assemblies in a partially rodded state.
\end{abstract}

KEYWORDS: Pin-by-pin TORT-TD, sub-channel CTF, coupled code, transient, neutron transport.

\section{INTRODUCTION}

The physics of BWRs has become more complicated in recent years because modern fuel assemblies exhibit new sophisticated design features, given by, e.g., part length fuel rods, strongly varying radial fuel enrichment, and Gadolinium bearing burnable absorber pins. The SVEA-96 Optima2 fuel assembly is an example of a complex BWR assembly design. As acceptance criteria are local parameters, the study of local phenomena requires the application of high-fidelity coupled multi-physics simulations at pin and sub-channel scale for accurate and realistic predictions of local safety parameters.

This paper describes the development of a 3-d pin-by-pin neutron transport and sub-channel thermal hydraulic model of a SVEA-96 Optima2 fuel assembly for the coupled code system TORT-TD/CTF which is described in the following section. Next, the characteristics of the SVEA-96 Optima2 assembly are outlined in section 3, followed by the description of the generation of pin cell-wise homogenized fewgroup cross section libraries using the lattice and depletion code HELIOS in section 4. The TORT-TD pin-by-pin model of SVEA-96 Optima2 and its qualification using Serpent are presented in sections 5. Section 6 describes the CTF sub-channel model of SVEA-96 Optima2. Finally, coupled steady-state and transient simulation results of a single assembly and a 4-by-4 mini core are presented in section 7. 


\section{TORT-TD/CTF - COUPLED SUB-CHANNEL THERMAL-HYDRAULICS/PIN-BY-PIN NEUTRON TRANSPORT CODE}

For the 3-d high-resolution thermal hydraulics/neutron kinetics simulations, the coupled code system TORT-TD/CTF [1] has been applied. In this code system, the thermal hydraulics is simulated by CTF, which is an advanced thermal-hydraulics sub-channel code. Originating from COBRA-TF (Coolant Boiling in Rod Arrays - Two Fluid), it is being developed and improved at the North Carolina State University (NCSU) in cooperation with the Oak Ridge National Laboratory (ORNL) and rebranded as CTF [2]. It is a part of the U.S. DOE CASL and EC NURESAFE projects. CTF uses a two-fluid, three-field (i.e. liquid film, liquid drops, and vapor) modelling approach. In the last decade, CTF has been extensively validated [3] for Pressurized Water Reactor (PWR), Boiling Water Reactor (BWR), VVER, Small Modular Reactor (SMR), and research reactor applications. The version used in this paper is CTF 3.7. The neutron kinetics is treated at pin-by-pin level by the transient 3-d few-group transport code TORT-TD developed at GRS [4]. TORT-TD solves the time-dependent 3D few-group fine-mesh transport equation in either discrete ordinates representation or diffusion approximation, both with arbitrary number of energy groups, arbitrary Legendre scattering expansion order and arbitrary number of delayed neutron precursor groups. Both codes are coupled using an explicit coupling scheme [1] wherein both codes are compiled into a single executable with a newly developed coupling supervisor. For steady state and transient simulations, CTF receives pin-wise and axially resolved power density distributions from TORT-TD, from which CTF calculates subchannel-resolved 3-d distributions of moderator and coolant density as well as pin-wise fuel temperature and transfers the respective distributions back to TORT-TD. TORT-TD takes these pin-wise and subchannel-wise, i.e. not assembly averaged, 3-d distributions for updating the pin-cell cross sections by interpolation in the cross section libraries. A coolant-centered subchannel representation (see Figure 1) is used in this paper.

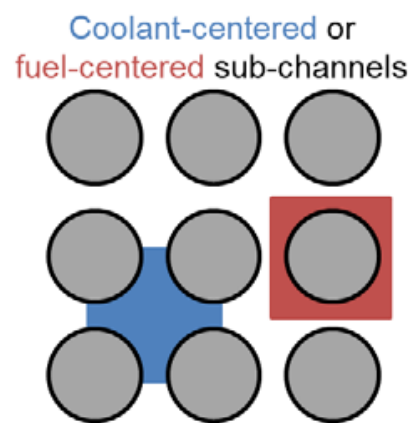

Figure 1. Schematic representation of a coolant-centered (blue, used in this paper) and a fuel rod-centered (red) CTF subchannel.

\section{SVEA-96 OPTIMA2 FUEL ASSEMBLY}

The SVEA-96 Optima2 fuel assembly consists of four sub-bundles separated by a cruciform internal structure (water cross) in the channel including a central canal. It features fuel rods with $1 / 3$ and $2 / 3$ active length, a not strictly regular pin lattice and four $\mathrm{Gd}_{2} \mathrm{O}_{3}$-bearing fuel rods in each sub-bundle with different Gd contents (see Figure 2). This quarter-channel design may have consequences on the void fraction distribution across the assembly, e.g., if it is subject to the influence of absorber blades or if there are different flow conditions between the four sub-bundles. 

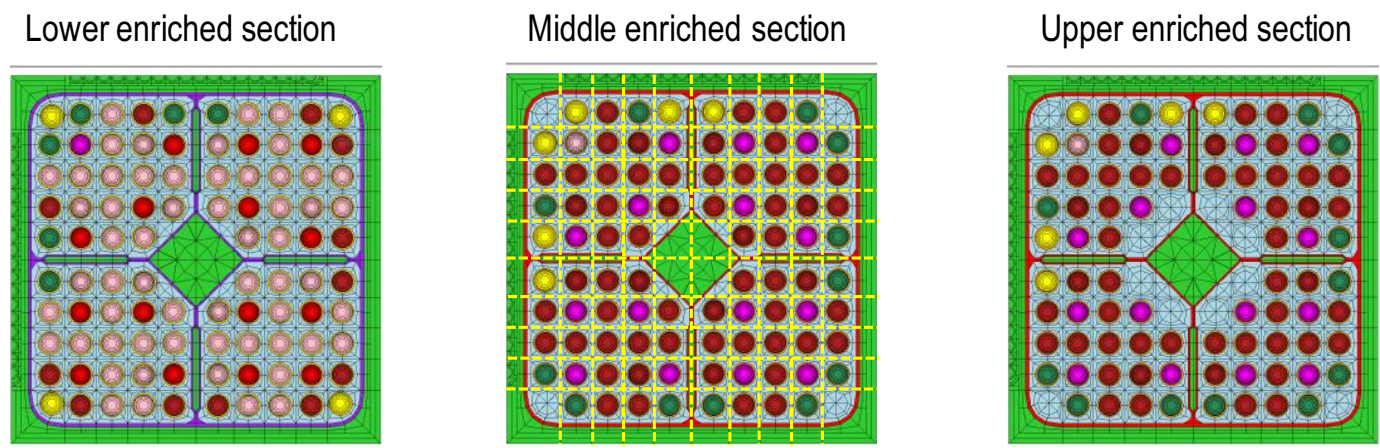

Figure 2. HELIOS models of the three enriched axial sections of the SVEA-96 Optima2 fuel assembly. Different colors of the pins represent different enrichments and different $\mathrm{Gd}_{2} \mathrm{O}_{3}$ contents. The dashed yellow grid in the middle indicates the $\mathbf{1 0 - b y - 1 0}$ pin cell lattice boundaries used for pin cell-wise cross section homogenization.

\section{GENERATION OF PIN-CELL HOMOGENIZED CROSS SECTION LIBRARIES}

The lattice and depletion code HELIOS-1.12 [5] is used for the generation of pin cell-homogenized macroscopic cross section libraries. HELIOS is a 2-d general geometry lattice and depletion code which solves the multi-group transport equation using the current coupling collision probability (CCCP) method. The HELIOS models for the individual axial sections of the SVEA-96 Optima2 are shown in Figure 2; the model for the natural uranium head (not shown here) is similar to the upper enriched section with all pins containing $0.71 \mathrm{wt} \%{ }^{235} \mathrm{U}$. Using the 10-by-10 lattice also shown in Figure 2, pin cell homogenized cross tabulated sections libraries in a NEMTAB-like format have been generated in eight energy groups (see Table 1), which were parameterized with respect to fuel temperature, channel water (light blue area in Figure 2) density and bypass water (green area in Figure 2) density, using a total of 156 library support points. For the fuel temperature, 6 equally spaced library support points between $500 \mathrm{~K}$ and $2000 \mathrm{~K}$ have been used. The potential range of the channel water density is accounted for by 13 equally spaced library support points between $0.03 \mathrm{~g} / \mathrm{cm}^{3}$ and $0.87 \mathrm{~g} / \mathrm{cm}^{3}$, whereas two support points $\left(0.72 \mathrm{~g} / \mathrm{cm}^{3}\right.$ and $0.8 \mathrm{~g} / \mathrm{cm}^{3}$ ) have been used to repersent the bypass water density.

Table 1. Eight energy groups structure used for the generation of pin cell homogenized cross sections.

\begin{tabular}{|c|c|c|c|c|c|c|c|}
\hline $\begin{array}{c}\text { Group } \\
\text { index }\end{array}$ & $\begin{array}{c}\text { Lower bound } \\
(\mathrm{eV})\end{array}$ & $\begin{array}{c}\text { Group } \\
\text { index }\end{array}$ & $\begin{array}{c}\text { Lower bound } \\
(\mathrm{eV})\end{array}$ & $\begin{array}{c}\text { Group } \\
\text { index }\end{array}$ & $\begin{array}{c}\text { Lower bound } \\
(\mathrm{eV})\end{array}$ & $\begin{array}{c}\text { Group } \\
\text { index }\end{array}$ & $\begin{array}{c}\text { Lower bound } \\
(\mathrm{eV})\end{array}$ \\
\hline 1 & $2.2313 \mathrm{E}+06$ & 3 & $9.1188 \mathrm{E}+03$ & 5 & $3.9279 \mathrm{E}+00$ & 7 & $1.4572 \mathrm{E}-01$ \\
\hline 2 & $8.2085 \mathrm{E}+05$ & 4 & $1.3007 \mathrm{E}+02$ & 6 & $6.2506 \mathrm{E}-01$ & 8 & $0.0000 \mathrm{E}+00$ \\
\hline
\end{tabular}

For the boundary pin cells facing the control blade, however, the pin cell shapes are not rectangular as can be seen in Figure 3 on the left. In order to have a regular pin cell lattice in TORT-TD, an approximate control blade model has been developed in which the circular absorber pin lattice is represented by absorber and cladding strips (see Figure 3, right). Whereas the length of absorber and cladding strips correspond to the dimension of the control blade, the absorber strip width $w$ has been iteratively adjusted such that the multiplication factor of the exact model is reproduced. Using two previous width iterates, $w_{i-2}$ and $w_{i-1}$, and their corresponding multiplication factors, $k_{i-2}$ and $k_{i-1}$, linear extrapolation is used to generate a better estimate $w_{i}$ :

$$
w_{i}=w_{i-2}-\frac{k_{\text {exact }}-k_{i-2}}{k_{i-1}-k_{i-2}}\left(w_{i-2}-w_{i-1}\right)
$$


where $k_{\text {exact }}$ is the multiplication factor of the model with the exact control blade. After 4 iterations, the reactivity difference between the approximate and the exact model is around $1 \mathrm{pcm}$.

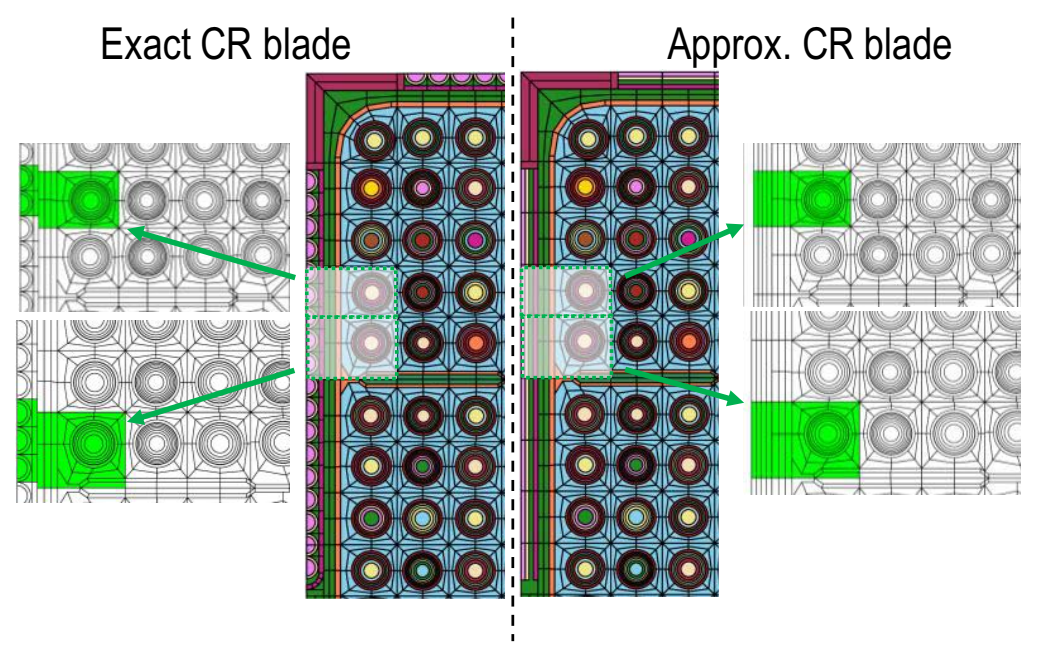

Figure 3. Exact CR blade model (left) and equivalent approximate CR blade model (right) for rectangular shapes of boundary pin cells.

Each of the four axial section consists of 100 pin cells. Accounting for uncontrolled and controlled states (with four possible orientations of the control blade), this results in a total of 3200 cross section libraries to describe the full SVEA-96 Optima2 fuel assembly on a pin-by-pin basis.

For the qualification of the HELIOS models, equivalent Monte-Carlo models for each axial section have been developed for Serpent [6] using ENDF/B-VII continuous energy nuclear data. Both multiplication factors and pin power distributions obtained with HELIOS have been compared to those calculated by Serpent. It is found that the multiplication factor obtained by HELIOS differs from the Serpent reference result by $18 \mathrm{pcm},-58 \mathrm{pcm}$ and $-194 \mathrm{pcm}$ for the lower, middle and upper axial zone of the fuel assembly, respectively. Regarding the pin-wise power distribution, the deviation of the HELIOS solution from the Serpent reference result is about $1.1 \%$ (RMS) with a maximum of $2.4 \%$. The observed differences between HELIOS and Serpent are compatible with similar studies found in literature [7].

\section{TORT-TD PIN-BY-PIN MODEL OF A SINGLE SVEA-96 OPTIMA2 ASSEMBLY}

Using the pin cell homogenized cross sections as described in the previous section, deterministic TORT-TD models of each of the four axial sections of the SVEA-96 Optima2 assembly with reflective boundary conditions have been developed first. For the qualification of these models, multiplication factors and pin power distributions obtained with TORT-TD have been compared to those calculated by the HELIOS models used to generate the cross sections. As shown in Table 2, the deviation between TORT-TD and HELIOS for both multiplication factor and pin power distribution is in the same range as when comparing HELIOS with Serpent. For both uncontrolled and controlled states, the RMS pin power relative deviations are around $1 \%$. While for the uncontrolled state, the maximum and minimum deviations are around $2 \%$ or below, for the controlled state, they are a little higher and range between $2.7 \%$ and $3.9 \%$. 
Table 2. TORT-TD multiplication factors as well as maximum, minimum and RMS deviations of the pin power distributions w.r.t. HELIOS for the middle zone at different burnup values.

\begin{tabular}{c|cc|ccc|cc|ccc} 
& \multicolumn{6}{|c|}{ Unrodded } & \multicolumn{6}{c}{ Rodded } \\
\hline Burnup & \multicolumn{2}{|c|}{ Multiplication factor } & \multicolumn{2}{|c|}{ Pin power rel. deviation } & Multiplication factor & \multicolumn{3}{c}{ Pin power rel. deviation } \\
\hline GWd/t & TORT-TD & HELIOS & Max. & Min. & RMS & TORT-TD & HELIOS & Max. & Min. & RMS \\
\hline 2.0 & 1.07787 & 1.07424 & $1.7 \%$ & $-1.2 \%$ & $0.9 \%$ & 0.869398 & 0.86942 & $3.9 \%$ & $-3.3 \%$ & $1.4 \%$ \\
\hline 5.0 & 1.16113 & 1.16245 & $1.4 \%$ & $-1.6 \%$ & $0.9 \%$ & 0.929048 & 0.93299 & $3.3 \%$ & $-3.6 \%$ & $1.2 \%$ \\
\hline 10.0 & 1.24848 & 1.25211 & $1.6 \%$ & $-2.2 \%$ & $1.2 \%$ & 0.991902 & 0.99791 & $2.7 \%$ & $-3.6 \%$ & $1.1 \%$ \\
\hline 30.0 & 1.08945 & 1.09316 & $1.4 \%$ & $-2.0 \%$ & $1.0 \%$ & 0.858687 & 0.86431 & $2.8 \%$ & $-3.7 \%$ & $1.1 \%$ \\
\hline 70.0 & 0.799138 & 0.80244 & $1.5 \%$ & $-1.5 \%$ & $0.8 \%$ & 0.617234 & 0.62136 & $3.0 \%$ & $-3.2 \%$ & $1.0 \%$
\end{tabular}

\section{CTF SUB-CHANNEL THERMAL HYDRAULIC MODEL}

A CTF model of the SVEA-96 Optima2 fuel assembly has been developed. In this model, only a quarter of the lower axial section (with 24 fuel rods) is modelled and applied to the overall fuel element height. The model contains 33 thermo-hydraulic channels and 24 fuel rod objects. A coolant-centered channel approach has been adopted. In the axial direction, 50 equidistant nodes are used. The shortened fuel rods are considered only through the axial power distribution. This quarter of a bundle model is used as a basic building block which can be multiplied and rotated as needed. For example, this model is copied 64 times in order to simulate the 4-by-4 minicore shown in section 7.2.

\section{TORT-TD/CTF SIMULATION RESULTS}

\subsection{Single-assembly Steady State Simulation}

Steady state coupled TORT-TD/CTF simulations of the SVEA-96 Optima2 fuel assembly in infinite lattice arrangement converged after 5 iterations (maximum relative change of local pin power between current and previous iteration below 1\%) between TORT-TD and CTF. Axial and radial distributions of pin power and moderator density have been investigated and appear physically plausible as shown in Figure 4.

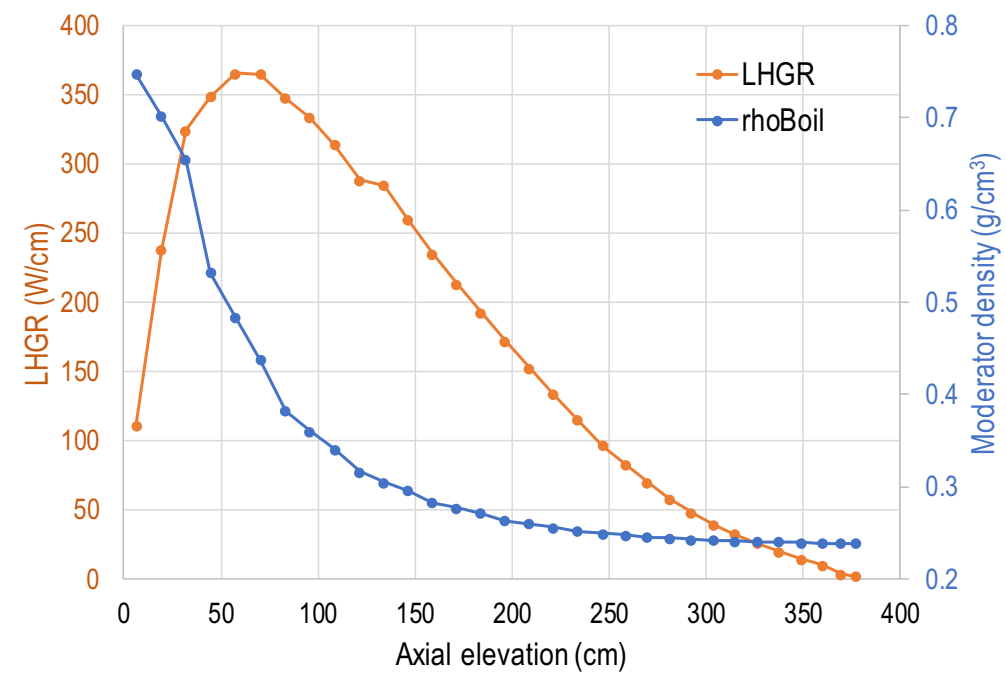

Figure 4. Radially averaged power density (orange) and moderator density (blue) axial profiles within a SVEA-96 Optima2 assembly (LHGR: linear heat generation rate). 
Corresponding visualizations of the pin power distributions of the middle axial section show that the pin power distribution is clearly peaked even for the uncontrolled state (see Figure 5, left panel). As the part length rods at the corners are missing, the maximum of the pin power is located at the next-to-corner rods. The relative differences between the power of the next-to-corner rods and its neighbors exceeds $20 \%$. It is shown that the presence of absorber blades leads to a strong radial tilt of the pin power distribution (see Figure 5, right panel), which results in different conditions between the sub-bundles of the same fuel assembly.
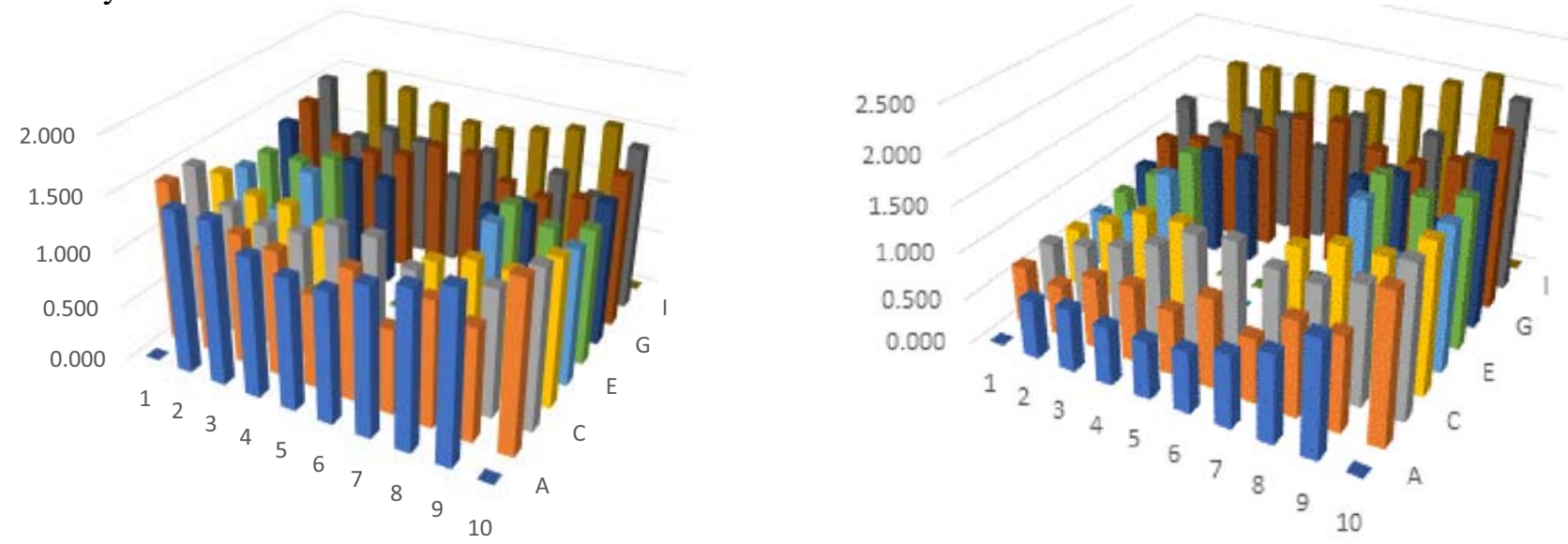

Figure 5. Normalized pin power distribution in the middle axial section of SVEA-96 Optima2 (left: unrodded, right: rodded) obtained with TORT-TD/CTF.

\subsection{Mini Core Steady State and Transient Simulations}

As a next step, a 4-by-4 mini core arrangement of SVEA-96 Optima2 fuel assemblies with different burnup states and a partially inserted control blade has been studied. The burnup states and locations of the control blades (all are withdrawn except for the one shown in red which is inserted $128 \mathrm{~cm}$ from the bottom) are given in Figure 6. The converged steady-state pin power distributions at two axial elevations (70.4 $\mathrm{cm}$ and $146.75 \mathrm{~cm}$ corresponding to the lower and middle axial section, respectively) are shown in Figure 7. Clearly, the local influence of the partially inserted control blade is seen as well as the locally reduced power (appearing in blue) at the $\mathrm{Gd}$ pin positions in the low-burnup $(0.35 \mathrm{GWd} / \mathrm{t})$ fuel assembly locations and the high local power generation (red) in the next-to-corner rods in the middle axial section. The corresponding moderator density distributions are shown in Figure 8. The simulation of a transient with TORT-TD/CTF initiated by a $12.5 \mathrm{~cm}$ withdrawal of the partially inserted control blade, starting at $0.2 \mathrm{~s}$ and ending at $0.3 \mathrm{~s}$, appears plausible as shown in Figure 9. In addition, coupled thermalhydraulic/neutron kinetic TORT-TD/CTF stability analyses have been performed for a single SVEA-96 Optima2 fuel assembly and the 4-by-4 miniciore (not shown here due to the lack of space).

\begin{tabular}{|c|c|c|c|}
\hline 14.0 & 36.0 & 28.0 & 30.0 \\
\hline 24.0 & 0.35 & 28.0 & 24.0 \\
\hline 14.0 & 40.0 & 0.35 & 38.0 \\
\hline 28.0 & 0.35 & 28.0 & 14.0 \\
\hline
\end{tabular}

Figure 6. 4-by-4 SVEA-96 Optima2 mini core configuration with burnup values (in GWd/t) and location of the control blades (red cross: partially inserted from the bottom, blue crosses: withdrawn). 


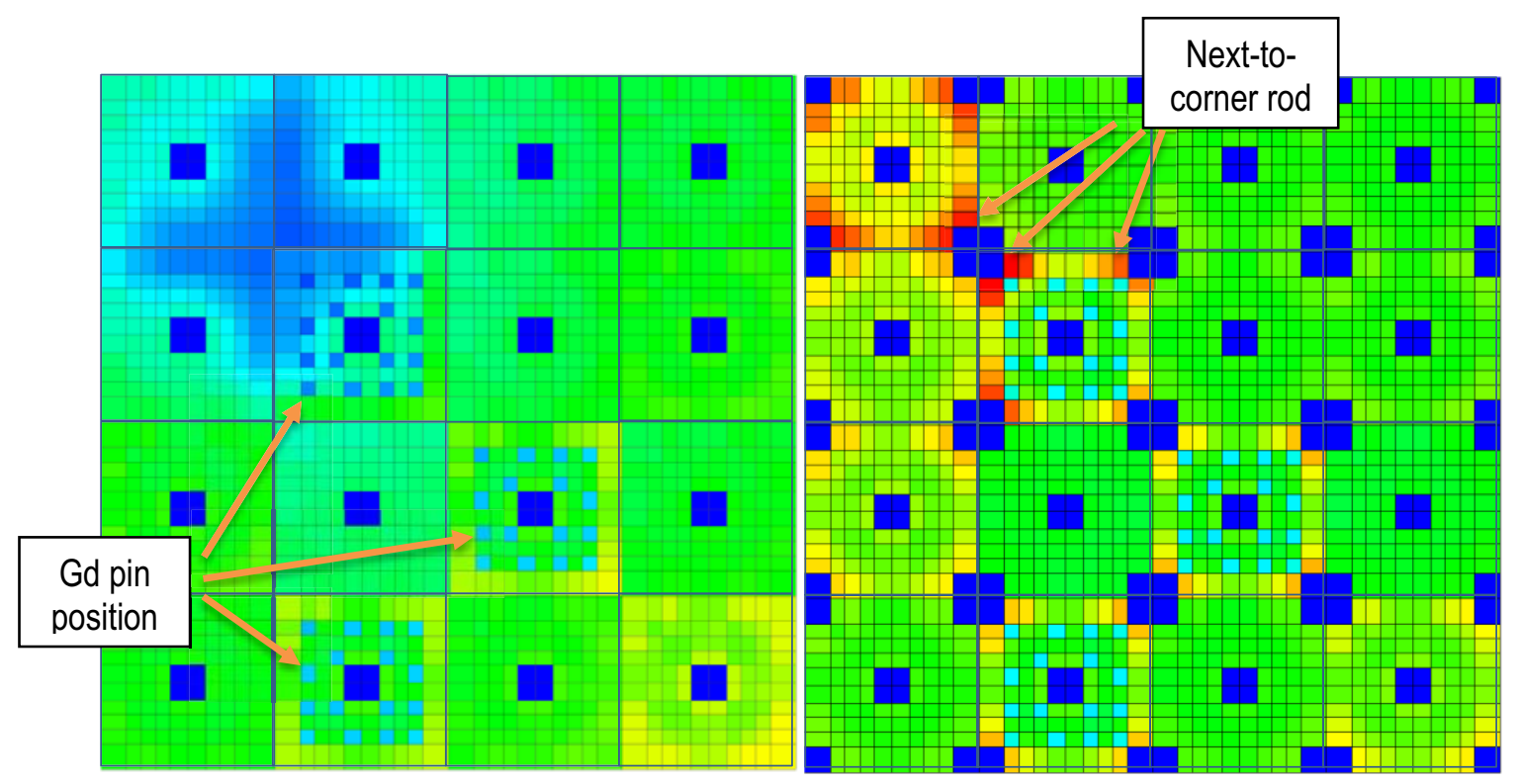

Figure 7. Steady-state TORT-TD/CTF pin power distributions in the 4-by-4 mini core model at axial positions $70.4 \mathrm{~cm}$ (left) and $146.75 \mathrm{~cm}$ (right). (blue: low, green: medium, red: high)

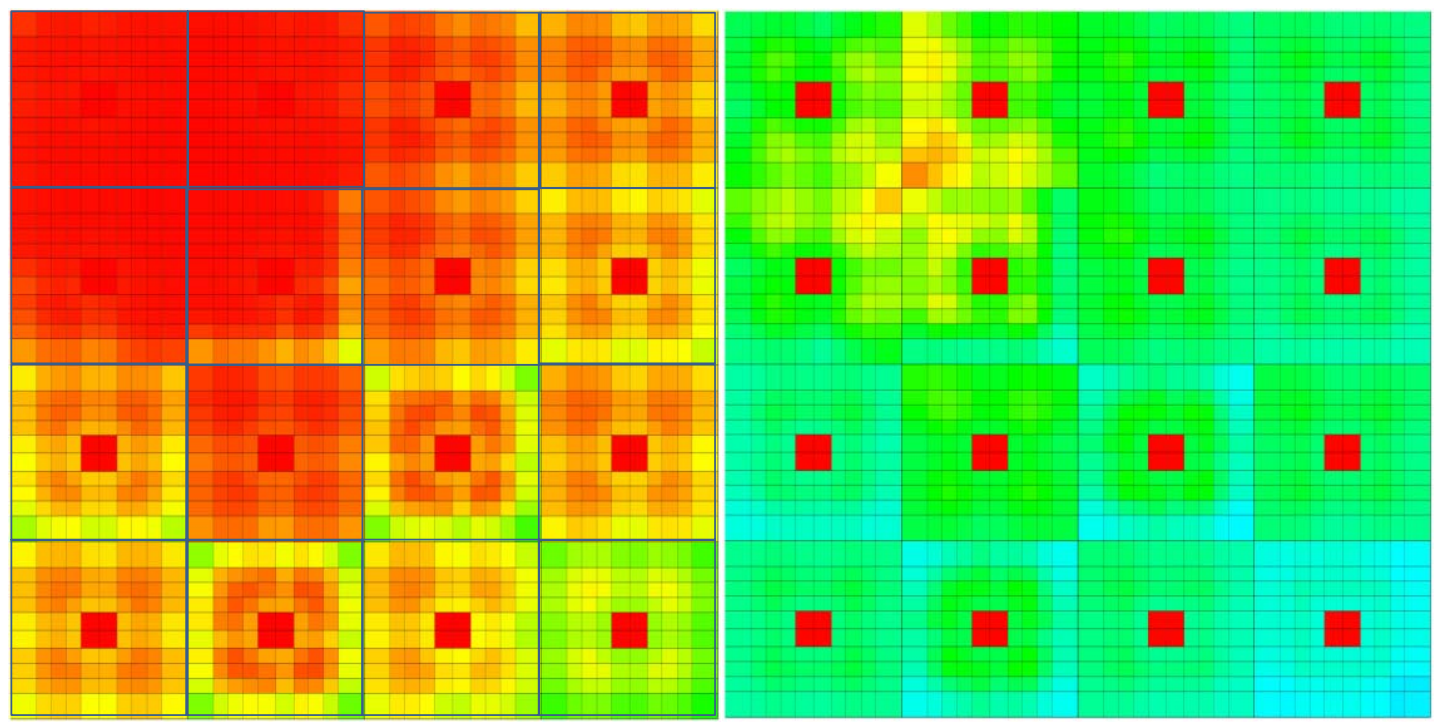

Figure 8. Same as Figure 7, but for the moderator density distribution. (blue: low, green: medium, red: high)

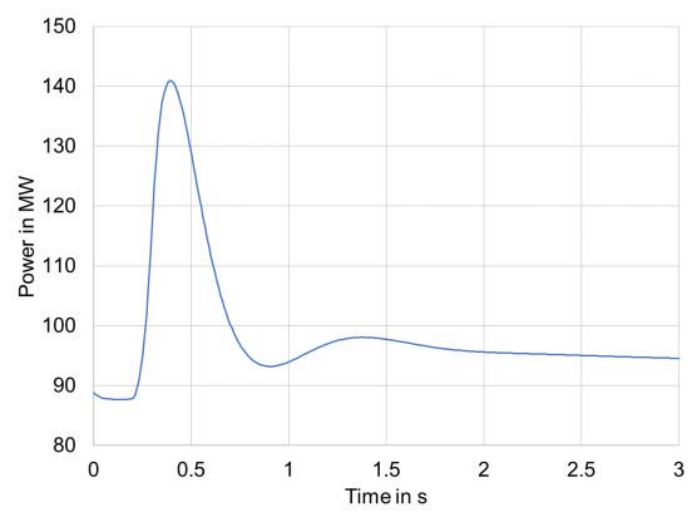

Figure 9. Transient response by TORT-TD/CTF of a $12.5 \mathrm{~cm}$ withdrawal of a partially inserted control blade (start at $0.2 \mathrm{~s}$ and end at $0.3 \mathrm{~s}$ ) in the lower axial section of one assembly in the 4-by-4 mini core configuration. 


\section{CONCLUSIONS}

In this paper, a 3-d pin-by-pin neutron transport and sub-channel thermal hydraulic model of a SVEA-96 Optima2 fuel assembly and a 4-by-4 minicore for the coupled code system TORT-TD/CTF using pin-cell homogenized parameterized cross section libraries generated by HELIOS are described. Coupled TORT-TD/CTF simulations of the single assembly and the minicore provide detailed information on local pin power and moderator density/void distributions. A transient simulation of a control blade withdrawal is also presented and appears physically plausible. With the 3-d coupled neutron kinetics and sub-channel thermal hydraulics code system TORT-TD/CTF, GRS has developed an advanced tool for high-fidelity multi-physics simulation of local neutron physical and thermal hydraulic phenomena in complex modern BWR assemblies and so provides a contribution to improved assessment of local safety parameters in LWR. Future work includes, on the one hand, improvements to the thermal-hydraulic model, in particular the explicit description of the part-length rods, which also necessitate extensions of the coupling interface. It is also envisaged to transfer the respective know-how and experience to future applications for the highfidelity safety assessment of local phenomena in innovative designs, e.g., liquid metal-cooled reactor systems including SMR.

\section{ACKNOWLEDGMENTS}

This work was supported by the German Federal Ministry for the Environment, Nature Conservation and Nuclear Safety.

\section{REFERENCES}

1. M. Christienne, M. Avramova, Y. Perin, A. Seubert, "Coupled TORT-TD/CTF Capability for HighFidelity LWR Core Calculations", International Conference on the Physics of Reactors (PHYSOR) Advances in Reactor Physics to Power the Nuclear Renaissance, May 9 - 14, 2010, Pittsburgh, Pennsylvania (USA).

2. M. Avramova et al., "CTF User's Manual", Technical report, Reactor Dynamics and Fuel Modeling Group, The North Carolina State University.

3. R. Salko et al., "CTF Validation and Verification", Technical report, Reactor Dynamics and Fuel Modeling Group, The North Carolina State University.

4. A. Seubert, K. Velkov, S. Langenbuch, ,The time-dependent 3D discrete ordinates code TORT-TD with thermal-hydraulic feedback by ATHLET models", International Conference on the Physics of Reactors (PHYSOR) - Nuclear Power: A Sustainable Resource, April 22 - 26, 2008, Interlaken (Switzerland).

5. J. J. Casal, R. J. Stamm'ler, E. A. Villarino, A. A. Ferri, "HELIOS: Geometric Capabilities of a New Fuel Assembly Program", International Topical Meeting on Advances in Mathematics and Computation, Pittsburgh (USA), 1991.

6. J. Leppänen, M. Pusa, T. Viitanen, V. Valtavirta, T. Kaltiaisenaho, "The Serpent Monte Carlo code, Status, development and applications in 2013", Annals of Nuclear Energy 82 (2015) 142-150.

7. K. Luszczek, "Validation and Benchmarking of Westinghouse Lattice Physics Methods, Examensarbete i Kärnenergiteknik 120 HP”, Avancerad Nivå, Stockholm, Sverige, 2015. 